This Health Hazard Evaluation (HHE) report and any recommendations made herein are for the specific facility evaluated and may not be universally applicable. Any recommendations made are not to be considered as final statements of NIOSH policy or of any agency or individual involved.

HETA 92-0357-2404

MARCH 1994

HENNEGAN COMPANY

\section{FLORENCE, KENTUCKY}

\section{SUMMARY}

On August 17, 1992, the National Institute for Occupational Safety and Health (NIOSH) received a management request for a health hazard evaluation (HHE) at Hennegan Company, Florence, Kentucky. The request concerned potential exposures to chemicals used in an ultraviolet (UV) coating process. No health complaints were reported. On November 24,1992 , an initial walkthrough survey was performed. Additional follow-up visits were made in December 1992 and October 1993, to perform environmental sampling for isopropanol, toluene, trimethyl benzene (TMB), total hydrocarbons, and trimethylolpropane triacrylate (TMPTA).

No TMPTA was detected in any of the general area or personal breathing-zone (PBZ) air samples. Assuming an average air sample volume of six liters, the Minimum Detectable Concentration (MDC) for TMPTA was 0.05 parts per million (ppm). Based on the results of the gas chromatography-mass spectrometry analysis of two of the charcoal tube field samples, isopropanol, toluene, trimethyl benzene isomers, and total hydrocarbons were selected for quantitation. The concentrations of these solvents were well below their respective occupational exposure criteria. For example, isopropanol was present in the highest concentrations among these four compounds, ranging from 15 to $58 \mathrm{ppm}$ in the five PBZ samples which were collected. These levels of isopropanol are below the NIOSH, the Occupational Safety and Health Administration (OSHA), and the American Conference of Governmental Industrial Hygienists (ACGIH) exposure criteria of $400 \mathrm{ppm}$ for an 8-hour time-weighted average (TWA). Toluene was not detected on any of the air samples. The highest level of TMB, $1.6 \mathrm{ppm}$, was measured on a PBZ sample collected on the second press person. This concentration, however, is still well below the NIOSH REL and OSHA PEL of $25 \mathrm{ppm}$ for this compound. All of the remaining PBZ samples collected for TMB measured only trace amounts. The total hydrocarbon levels (as ndecane) ranged from 0.28 to $1.9 \mathrm{ppm}$. No occupational exposure criteria exist for total hydrocarbons.

No health hazards were documented from airborne exposure to chemicals used in the UV-coating process on the web press. However, the hazard communication program at Hennegan should be improved. Also, an eye protection program should be instituted and enforced to prevent eye trauma from foreign bodies and chemical splashes. A noise evaluation should be conducted to determine current noise levels and the necessity of implementing a complete hearing conservation program. Other health and safety problems identified during this evaluation included inadequate emergency eye wash stations, consuming food, drink, or tobacco products in production areas, and developing a written respiratory protection program.

Keywords: SIC 2754 (Commercial Printing, Gravure), isopropanol, toluene, total hydrocarbons, trimethylolpropane triacrylate, ultraviolet-cured inks, printing, skin sensitization 


\section{Page 2 - Health Hazard Evaluation Report No. 92-0357}

\section{INTRODUCTION}

On August 17, 1992, the National Institute for Occupational Safety and Health (NIOSH) received a management request to conduct a health hazard evaluation (HHE) at Hennegan Company in Florence, Kentucky. The request concerned potential exposures to chemicals used in the ultraviolet (UV) coating process on the web press. No health complaints were reported.

On November 24, 1992, an initial site visit was performed. The site visit consisted of a walkthrough survey, along with an assessment of the industrial hygiene and safety conditions, and work practices. Additional follow-up visits were made in December 1992 and October 1993, to perform environmental sampling.

\section{BACKGROUND}

Hennegan Company is a print shop which produces posters, catalogs, magazines, annual reports, labels, displays, and art prints. Hennegan Company, established in 1886, has facilities in both Cincinnati, Ohio, and Florence, Kentucky, and employs approximately 300 workers over three shifts. Of these, 135 are production workers at the Florence site. Approximately $75 \%$ of the work force is male.

Hennegan's production facility in Florence is housed in an one-story building. The 90,000 square feet of production area includes two web-fed printing presses, one sheet-fed printing press, and bindery operations.

Based on the information provided in the request and in the opening conference, the investigation focused on the area in which the web-fed press which has the UV curing process. A general process description of this area is presented below.

\section{Web-feed Press}

Rolls of paperboard are delivered and aligned within the web-fed printing press. The press prints up to six colors on the paperboard prior to passing through the drying oven, which is heated to $250^{\circ} \mathrm{F}$. The paperboard then proceeds to the chiller before the UV-cured coating is applied. Once the UV-cured ink is coated onto the paperboard, it is cured by the UV light, and either folded into signatures or cut into sheets and stacked for distribution.

A five person crew operates the web-fed press. The press is cleaned after every run. Nitrile butyl gloves, Tyvek® suits, face shields, goggles, and respirators are available to the workers.

\section{ENVIRONMENTAL METHODS}

The environmental evaluation focused primarily on air monitoring, but also included the collection of bulk samples, and walk-through evaluations of environmental and safety conditions. Environmental monitoring included evaluation of exposures to trimethylolpropane triacrylate (TMPTA), isopropanol, toluene, perchloroethylene, total hydrocarbons, and trimethyl benzene.

\section{Trimethylolpropane Triacrylate}

Personal breathing zone (PBZ) and area air samples for TMPTA were collected on XAD-7 tubes according to the Occupational Safety and Health Administration (OSHA) "stop-gap" method. Presently, this method has not been validated and has desorption efficiencies which are slightly less $(69.7 \% \pm 6.4)$ than the minimum acceptable value of $75 \%$.

Eight-hour time-weighted average (TWA) samples were connected via Tygon ${ }^{\circledR}$ tubing to Gillian Lo Flow Sampler@ battery-operated personal sampling pumps. Air was sampled through the 


\section{Page 3 - Health Hazard Evaluation Report No. 92-0357}

tubes at a nominal flow rate of 0.02 liters per minute ( $\ell / \mathrm{min})$. After sampling, the XAD-7 tubes were removed and desorbed with 2 milliliters (ml) of methanol. The samples were analyzed using high performance liquid chromatography (HPLC) using an UV detector. The analytical limit of detection (LOD) for this method is 2 micrograms $(\mu \mathrm{g})$ per sample.

\section{Organic Solvents}

Bulk, area, and PBZ air samples for organic solvents were collected using charcoal tubes as the collection media. The charcoal tubes were connected via Tygon ${ }^{\circledR}$ tubing to battery-operated personal sampling pumps. Air was sampled through the tubes at a nominal flow rate of 0.02 l/min.

After sampling, the charcoal tubes were removed and desorbed in carbon disulfide. Two bulk air samples were qualitatively analyzed for organic compounds using gas chromatography-mass

\begin{tabular}{||c|c|c||}
\hline Compound & $\begin{array}{c}\text { Limit of } \\
\text { Detection }\end{array}$ & $\begin{array}{c}\text { Limit of } \\
\text { Quantitation }\end{array}$ \\
\hline Toluene & $3 \mu \mathrm{g} / \mathrm{sample}$ & $10 \mu \mathrm{g} / \mathrm{sample}$ \\
\hline Isopropanol & $3 \mu \mathrm{g} / \mathrm{sample}$ & $9 \mu \mathrm{g} / \mathrm{sample}$ \\
\hline $\mathrm{TMB}$ & $11 \mu \mathrm{g} / \mathrm{sample}$ & $35 \mu \mathrm{g} / \mathrm{sample}$ \\
\hline Total HC & $3 \mu \mathrm{g} / \mathrm{sample}$ & $10 \mu \mathrm{g} / \mathrm{sample}$ \\
\hline $\begin{array}{c}\text { Abbreviations: } \\
\text { TMB } \\
\text { Total HC } \\
\mu \mathrm{g} / \mathrm{sample}\end{array}$ & $\begin{array}{l}=\text { Trimethyl } \\
\text { benzene isomers } \\
=\text { Total } \\
\text { hydrocarbons } \\
=\text { micrograms per } \\
\text { sample }\end{array}$ \\
\hline \hline
\end{tabular}

spectrometer (GC-MS). Based on the results of the bulk samples, samples were quantitatively analyzed for isopropanol, toluene, trimethyl benzene, and total hydrocarbons using a combination of NIOSH Methods 1400 and $1500^{1}$, with modifications. The quantitative samples were analyzed using gas chromatography-flame ionization detection (GC-FID). The analytical LOD for these four compounds using these NIOSH Methods are listed in the adjacent chart.

All of the Gillian Lo Flow Sampler® pumps were calibrated prior to and after sampling using the Gillian Gilibrator ${ }^{\circledR}$. The Gillian Gilibrator ${ }^{\circledR}$ was calibrated against a primary standard. For subsequent calculations of sample volumes, the mean pre- and post flow rates were used. A minimum of $10 \%$ of the sampled XAD-7 and charcoal tubes were prepared and submitted as field blanks with the sample sets.

\section{EVALUATION CRITERIA}

As a guide to the evaluation of the hazards posed by workplace exposures, NIOSH field staff employ evaluation criteria for the assessment of a number of chemical (and physical) agents. The primary sources of environmental evaluation criteria for the workplace are the following: (1) NIOSH Criteria Documents and Recommended Exposure Limits (RELs), (2) the U.S. Department of Labor, OSHA Permissible Exposure Limits (PELs), and (3) the American Conference of Governmental Industrial Hygienists' (ACGIH) Threshold Limit Values ${ }^{\circledR}$ (TLVs). ${ }^{1,2,3}$ The objective of these criteria for chemical agents is to establish levels of inhalation 


\section{Page 4 - Health Hazard Evaluation Report No. 92-0357}

exposure to which the vast majority of workers may be exposed without experiencing adverse health effects.

Full-shift and shorter duration inhalation criteria are available depending on the specific physiologic properties of the chemical substance. Full-shift limits are based on the timeweighted average (TWA) airborne concentration of a substance that most workers may be repeatedly exposed to during a normal 8 or 10-hour day, up to 40 hours per week for a working lifetime, without adverse effect. Some substances have recommended short-term exposure limits (STELs) or ceiling limits which are intended to supplement the full shift criteria where there are recognized irritative or toxic effects from brief exposures to high airborne concentrations. STELs are based on TWA concentrations over 15 minute time periods, whereas ceiling limits are concentrations which should not be exceeded even momentarily.

Occupational health criteria are established based on the available scientific information provided by industrial experience, animal or human experimentation, and epidemiological studies. Differences between the NIOSH RELs, OSHA PELs, and the ACGIH TLVs® may exist because of different scientific philosophy and interpretations of technical information. When comparing the exposure criteria, it should be noted that employers are legally required to meet those levels (and any conditions) specified by an OSHA PEL. The legal rulemaking process for promulgation of OSHA PELs is an arduous and time consuming task and the OSHA PELs may be required to take into account the technical and economical feasibility of controlling exposures in various industries where the agents are used. Hence, OSHA PELs may not be established based on the most current scientific information. In contrast, the NIOSH RELs are primarily based upon the prevention of occupational disease without assessing the economic feasibility of the affected industries and as such tend to be very conservative. ACGIH is not a governmental agency; it is a professional organization whose members are industrial hygienists or other professionals in related disciplines and are employed in the public or academic sector. TLVs ${ }^{\circledR}$ are developed by consensus agreement of the ACGIH TLV® committee and are published annually. The documentation supporting the TLVs ${ }^{\circledR}$ (and proposed changes) is periodically reviewed and updated if believed necessary by the committee. It is not intended by ACGIH for TLVs ${ }^{\circledR}$ to be applied as the threshold between safe and dangerous inhalation exposure.

It is important to note that not all workers will be protected from adverse health effects if their exposures are maintained below these occupational health exposure criteria. A small percentage may experience adverse health effects because of individual susceptibility, a pre-existing medical condition, previous exposures, and/or a hypersensitivity (allergy). In addition, some hazardous substances may act in combination with other workplace exposures, or with medications or personal habits of the worker (such as smoking, etc.) to produce health effects even if the occupational exposures are controlled to the limit set by the evaluation criterion. These combined effects are often not considered by the chemical specific evaluation criteria. Furthermore, many substances are appreciably absorbed by direct contact with the skin and thus potentially increase the overall exposure and biologic response beyond that expected from inhalation alone. Finally, evaluation criteria may change over time as new information on the toxic effects of an agent become available. Because of these reasons, it is prudent for an employer to maintain worker exposures well below established occupational health criteria.

\section{Isopropanol (Isopropyl Alcohol)}

Isopropanol is a colorless, volatile, flammable liquid of low toxicity that is used as a chemical intermediate, as a general purpose solvent, and is present in skin lotions, cosmetics, and pharmaceuticals. ${ }^{4,5}$ The vapor of isopropanol is irritating to the eyes and mucous membranes; inhalation of high concentrations can cause depression of the central nervous system. ${ }^{4,5}$ The potential effects from dermal contact with the liquid are insignificant; cutaneous absorption should not contribute to systemic toxicity, and generally does not produce skin irritation, except with hypersensitive individuals. ${ }^{5}$ 


\section{Page 5 - Health Hazard Evaluation Report No. 92-0357}

The inhalation exposure criteria established for isopropanol by NIOSH, OSHA, and ACGIH are equivalent -- a full-shift TWA of $400 \mathrm{ppm}$, and a 15-minute STEL of $500 \mathrm{ppm} .^{1,2,3}$

\section{Trimethylolpropane Triacrylate (TMPTA)}

TMPTA is one of several multifunctional acrylates used in UV-cured inks. In use since the early 1970 's, these acrylates, which act as cross-linkers and diluents, quickly polymerize following exposure to UV. ${ }^{6}$ While these substances have revolutionized the printing industry, the uncured inks may contain constituents (such as TMPTA or other acrylates) which are skin irritants or sensitizers. For example, studies have shown workers developing dermatitis of the hands and skin following direct skin contamination with TMPTA-containing inks. ${ }^{7}$ Allergic contact dermatitis from UV-curing acrylate used in the manufacture of optical fibers has also been documented. ${ }^{8}$

NIOSH, OSHA, and the ACGIH have not established occupational exposure criteria for TMPTA.

\section{Trimethyl Benzene (TMB)}

The three isomers of TMB are used in gasoline, various solvents, and as a UV stabilizer in plastics. Since the pharmacological and toxicological properties of the three isomers are similar, the exposure criteria is applicable for the mixture. TMB is an eye, nose, and respiratory irritant. Liquid TMB is a skin irritant but systemic intoxication due to absorption through the skin is not probable. ${ }^{9}$

The inhalation exposure criteria established for TMB by NIOSH and the ACGIH are equivalent - a full-shift TWA of 25 ppm. ${ }^{1,3}$ Currently there is no OSHA PEL for this compound.

\section{RESULTS}

\section{Trimethylolpropane Triacrylate (TMPTA)}

No TMPTA was detected in any of the general area or PBZ air samples collected as part of this evaluation. Assuming an average sample volume of six liters, the Minimum Detectable Concentration (MDC) for this sample set was $0.05 \mathrm{ppm}$.

\section{Organic Solvents}

Based on the results of the gas chromatography-mass spectrometry analysis of two of the charcoal tube field samples, isopropanol, toluene, trimethyl benzene isomers, and total hydrocarbons were selected for quantitation. As shown in Table 1, the concentrations of these solvents were well below their respective occupational exposure criteria.

Isopropanol was present in the highest concentrations among these four compounds, ranging from 15 to $58 \mathrm{ppm}$ in the five PBZ samples which were collected. These levels of isopropanol are below the NIOSH, OSHA, and ACGIH exposure criteria of $400 \mathrm{ppm}$ for an 8-hour TWA.

The highest level of TMB, $1.6 \mathrm{ppm}$, was measured on a PBZ sample collected the second press person. This concentration, however, is still well below the NIOSH REL and OSHA PEL of 25 ppm for this compound. All of the remaining PBZ samples collected for TMB measured only trace amounts.

Toluene was not detected on any of the air samples. The total hydrocarbon levels (as n-decane) ranged from 0.28 to $1.9 \mathrm{ppm}$. No occupational exposure criteria exist for total hydrocarbons. 


\section{Page 6 - Health Hazard Evaluation Report No. 92-0357}

\section{DISCUSSION}

Although the concentrations of isopropanol, TMB, and total hydrocarbons measured during this survey do not exceed OSHA, NIOSH, or ACGIH occupational exposure criteria, the low levels of volatile organic compounds (VOCs) may still present some risk of irritation in certain more susceptible individuals. For example, indoor environmental quality studies (typically in nonindustrial work settings such as offices) have measured wide ranges of VOC concentrations in indoor air as well as differences in the mixtures of chemicals which are present. Research also suggests that the irritant potency of these VOC mixtures can vary. Some researchers have compared VOC levels with human responses (such as headache and irritative symptoms of the eyes, nose, and throat). Research conducted in Europe suggests that complaints by building occupants may be more likely to occur when VOC concentrations increase. However, neither NIOSH nor OSHA currently have specific exposure criteria for low-level VOC mixtures in the non-industrial (or industrial) environment. It should also be emphasized that the highly variable nature of these complex VOC mixtures can greatly affect their irritancy potential. Finally, much of the research which has been conducted has examined non-industrial work areas such as offices and schools and, as such, may not be directly applicable to occupational environments such as those at Hennegan.

Although TMPTA was not detected in any of the general area and PBZ air samples collected at part of this evaluation, it is still appropriate that personal protective clothing be used during the press clean-up. Skin contact with this acrylate, or with the other solvents used at Hennegan, can cause dermatitis.

\section{RECOMMENDATIONS}

1. Eye trauma from foreign bodies and splashes are preventable. Hennegan should institute and enforce a program requiring the use of eye protection. Safety glasses should be required in all production areas and chemical splash goggles should be required whenever chemicals are being transferred from one container to another.

2. Appropriate personal protective clothing should be used during the press clean-up. Skin contact with solvents can cause dermatitis.

3. Labeling of chemicals, worker training, and other aspects of the hazard communication should be improved. Hennegan is required to meet all provisions of the hazard communication standard (29 CFR 1910.1200) as set forth by OSHA. Chemical containers should be labeled. The labels should identify the contents and any known hazards that are associated with that material.

4. Solvents used throughout Hennegan contain flammable liquids which are regulated by OSHA, primarily under 29 CFR 1910.106. During the investigation, it was observed that the flammable solvents were transferred into unapproved containers, and solvent-drenched rags were stored in open containers. Hennegan is required to use containers of the appropriate design, construction, and capacity as set forth by the OSHA standard 1910.106 for flammable and combustible liquids.

5. Although a noise survey was not conducted as part of this survey, there were a number of deficiencies noted regarding the requirements in the OSHA noise standard (29 CFR 1910.95). Periodic noise surveys, which include both sound level measurements and dosimetry, should be performed to determine the noise levels. Signs should be posted to distinguish the areas where hearing protection is required. Hennegan should require the use of hearing protection devices in areas which exceed a TWA noise level of 85 decibels on an A-weighted scale. Workers should be trained on the effects of noise exposure and hearing loss, and encouraged to reduce both occupational and recreational noise to prevent noise- 


\section{Page 7 - Health Hazard Evaluation Report No. 92-0357}

induced hearing loss. The noise standard requires employee notification of both the sampling and audiometric results.

6. If respirators are available to workers, Hennegan must meet the minimum requirements set forth by the U.S. Department of Labor, OSHA in the respiratory protection standard (29 CFR 1910.134), which includes written standard operating procedures, medical surveillance, fit-testing, worker training, and all other aspects of the program. ${ }^{2}$

7. Safety equipment, including eye wash stations and safety showers, should be installed and readily accessible as set forth by the OSHA standard 29 CFR 1910.151. ${ }^{2}$ The current portable eyewash system should be replaced with eye wash facilities which have a minimum of 15 minutes flushing time.

8. Ingestion resulting from hand-to-mouth contact with contaminated food, cigarettes, and other objects is another route of worker exposure to toxic substances. Workers should be prohibited from consuming food, drink, or tobacco products in the production area. Also, after leaving the production area, workers should wash their hands prior to eating, drinking, or smoking.

9. Since flammable solvents are used in the printing process, Hennegan should prohibit smoking in the production area. This will reduce the risk of a fire by removing a source of ignition.

10. NIOSH recommends that the use of tobacco products be curtailed in situations where employees may be exposed to chemical substances which may interact with tobacco products and where non-smoking workers may be exposed to side-stream cigarette smoke. The best method for controlling worker exposure to tobacco smoke is to eliminate smoking from the workplace. Until this is achieved, smoking should be restricted to areas outside of the plant or to a designated area such as a smoking room which has additional dedicated ventilation. The air from this smoking area should be exhausted directly to the outside and not recirculated within the building. ${ }^{10}$

\section{REFERENCES}

1. CDC [1988]. NIOSH recommendations for occupational safety and health standards 1988. Atlanta, GA: U.S. Department of Health and Human Services, Public Health Service, Centers for Disease Control, National Institute for Occupational Safety and Health. M M WR $\underline{37}$ (supp. S-7).

2. Code of Federal Regulations [1989]. 29 CFR 1910.1000. Washington, DC: U.S. Government Printing Office, Federal Register.

3. ACGIH [1992]. Threshold limit values and biological exposure indices for 1992-1993. Cincinnati, $\mathrm{OH}$ : American Conference of Governmental Industrial Hygienists.

4. ACGIH [1986]. Documentation of threshold limit values and biological exposure indices for chemical substances and physical agents. Cincinnati, $\mathrm{OH}$ : American Conference of Governmental Industrial Hygienists.

5. NIOSH [1976]. Criteria for a recommended standard: occupational exposure to isopropyl alcohol. Cincinnati, OH: U.S. Department of Health, Education, and W elfare, Public Health Service, Center for Disease Control, National Institute for Occupational Safety and Health, DHEW (NIOSH) Publication No. 76-142. 


\section{Page 8 - Health Hazard Evaluation Report No. 92-0357}

6. Bjorkner B [1984]. The sensitizing capacity of multifunctional acrylates in the guinea pig. Contact Dermatitis, Vol. 11:236-246.

7. Garabran DH [1985]. Dermatitis from aziridine hardener in printing ink. Contact Dermatitis, Vol. 12:209-212.

8. Maurice PD, Rycroft RJ G [1986]. Allergic contact dermatitis from UV-curing acrylate in the manufacture of optical fibers. Contact Dermatitis, Vol. 15:91.

9. Proctor NH, Hughes J P, Fischman M L [1988]. Chemical hazards of the w orkplace. 2nd ed. Philadelphia, PA: J.B. Lippincott.

10. NIOSH [1991]. Current intelligence bulletin 54: environmental tobacco smoke in the w orkplace, lung cancer and other health effects. Cincinnati, OH: U.S. Department of Health and Human Services, National Institute for Occupational Safety and Health, DHHS Publication No. 91-108.

\section{AUTHORSHIP AND ACKNOWLEDGEMENTS}

Survey Conducted By:

Report Prepared By:

Field Assistance by:

Originating Office:
Teresa M. Buchta, M.S. Industrial Hygienist Industrial Hygiene Section

Teresa M. Buchta, M.S. Industrial Hygienist Industrial Hygiene Section

Gregory A. Burr, CIH

Supervisory Industrial Hygienist Industrial Hygiene Section

Alan Echt, CIH

Industrial Hygienist Industrial Hygiene Section

Hazard Evaluations and Technical Assistance Branch Division of Surveillance, Hazard Evaluations and Field Studies 
Page 9 - Health Hazard Evaluation Report No. 92-0357

\section{DISTRIBUTION AND AVAILABILITY OF REPORT}

Copies of this report may be freely reproduced and are not copyrighted. Single copies of this report will be available for a period of 90 days from the date of this report from the NIOSH Publications Office, 4676 Columbia Parkway, Cincinnati, Ohio 45226. To expedite your request, include a self-address mailing label along with your written request. After this time, copies may be purchased from the National Technical Information Service, 5285 Port Royal Road, Springfield, VA 22161. Information regarding the NTIS stock number may be obtained from the NIOSH Publications Office at the Cincinnati address.

Copies of this report have been sent to:

1. The Hennegan Company

2. Graphic Communication International Union, Local 508

3. OSHA Region V

For the purpose of informing affected employees, copies of this report shall be posted by the employer in a prominent place accessible to the employees for a period of $\mathbf{3 0}$ calendar days. 
Table 1

Results of Personal Breathing-Zone and Area Air Samples for Isopropanol, Trimethyl Benzene, Toluene, and Total Hydrocarbons Sampling Location: Hennegan Company, Florence, Kentucky

Sampling Date: 10/8/93

HETA 92-0357

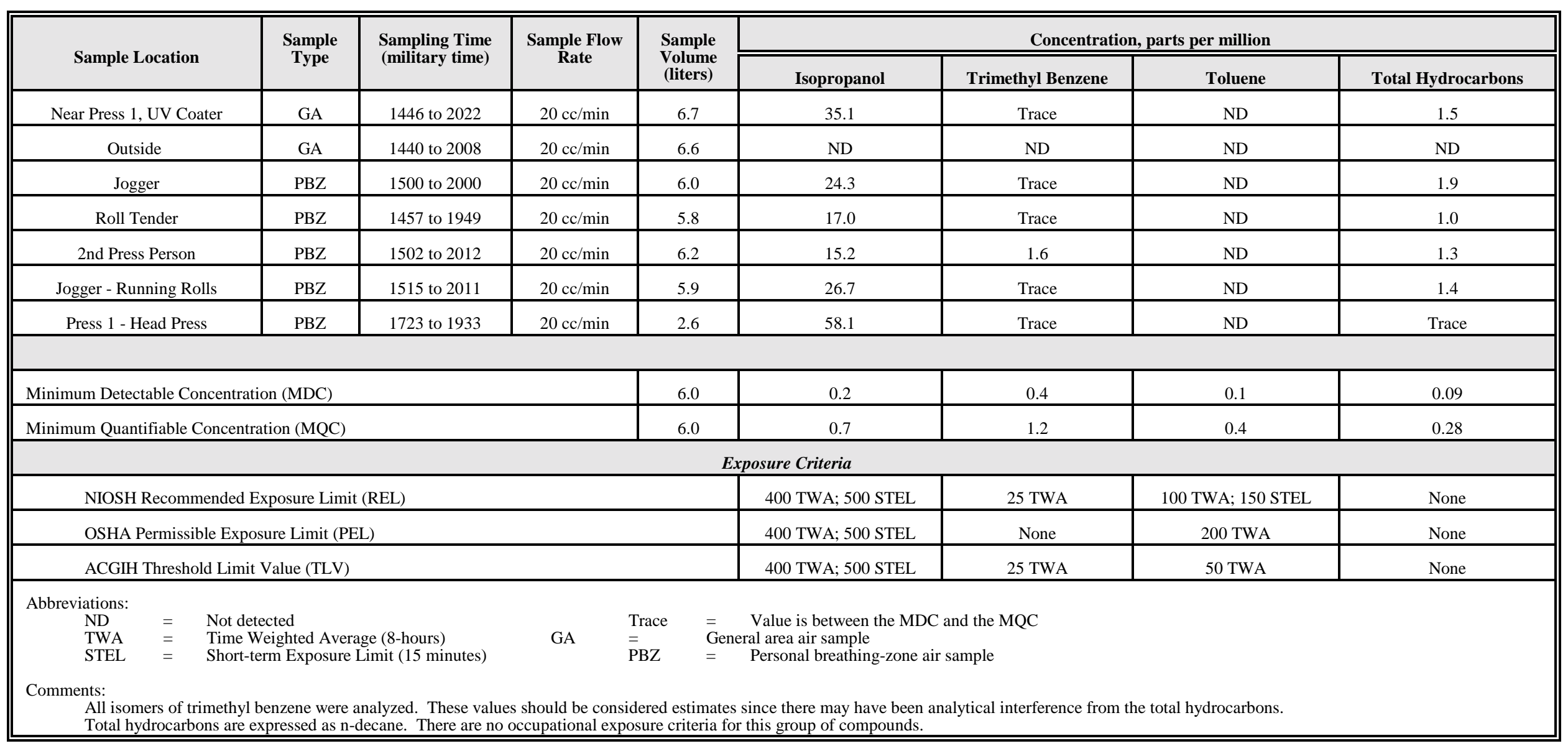

